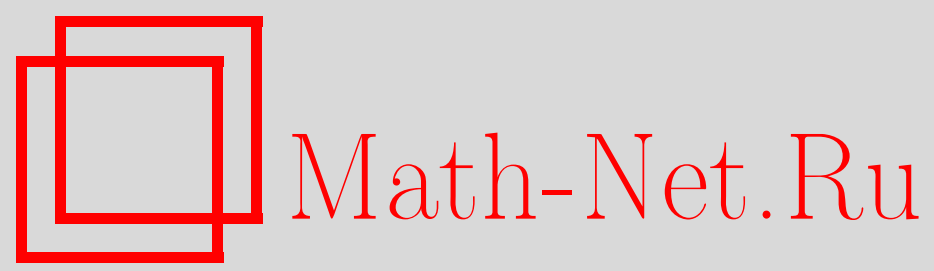

С. В. Полин, Перестановочные решетки отношений эквивалентности на декартовых произведениях и согласованные с ними системы уравнений. II, Maтем. вопр. криптогр., 2017, том 8, выпуск 3, 85-108

DOI: https://doi.org/10.4213/mvk233

Использование Общероссийского математического портала Math-Net.Ru подразумевает, что вы прочитали и согласны с пользовательским соглашением

http://www.mathnet.ru/rus/agreement

Параметры загрузки:

IP: 3.80 .253 .173

26 апреля 2023 г., 15:48:51 
МАТЕМАТИЧЕСКИЕ ВОПРОСЫ КРИПТОГРАФИИ

2017 T. 8 № 3 C. 85-108

$519.212 .2+519.115$

\title{
Перестановочные решетки отношений эквивалентности на декартовых произведениях и согласованные с ними системы уравнений. II
}

\author{
С. В. Полин
}

Академия криптографии Российской Федеращчи, г. Москва

Получено 20.IV.2015

Аннотация. Описаны введенные автором ранее $G A$-решетки и соответствующие им просто решаемые системы уравнений.

Ключевые слова: системы уравнений, решетки отношений

Permutation lattices of equivalence relations on the Cartesian products and systems of equations concordant with these lattices. II

\section{S. V. Polin}

Academy of Cryptography of the Russian Federation, Moscow

Abstract. A description of GA-lattices previously introduced by the author is given and easily solved systems of equations concordant with these lattices are presented.

Keywords: systems of equations, lattice of relations 
Настоящая работа является продолжением [12] и использует введенные в ней обозначения. Нумерация разделов, утверждений и формул продолжает нумерацию указанной работы. Ссылки вида «теорема 1.1», «лемма 2.1», «равенство (3.2)» являются ссылками на соответствующие утверждения и формулы работы [12].

\section{4. Условие неразложимости $G A$-решеток}

Вернемся к рассмотрению общего случая множества $A=\prod_{i=1}^{n} A_{i}$ и $G A$-решетки $\mathbf{L}_{A}$ отношений эквивалентности на множестве $A$.

Для произвольного подмножества $V \subset Z_{n}$ через $V^{\{2\}}$ обозначим множество всех подмножеств множества $V$, состоящих из двух различных элементов. Другими словами, $V^{\{2\}}$ состоит из всех пар $\left\{v_{1}, v_{2}\right\}$, где $v_{1}, v_{2} \in V$ и $v_{1} \neq v_{2}$. При этом пары $\left\{v_{1}, v_{2}\right\}$ и $\left\{v_{2}, v_{1}\right\}$ считаются одинаковыми.

В соответствии с теоремой 1.1 для всех пар $\left\{i_{1}, i_{2}\right\}$ определена $G A$-решетка

$$
\mathbf{L}_{\left\{i_{1}, i_{2}\right\}}=\left\langle\theta \in \mathcal{E}\left(B^{2}\right) \mid \zeta_{\left\{i_{1}, i_{2}\right\}}(\theta) \in \mathbf{L}_{A}\right\rangle
$$

отношений эквивалентности на множестве $A_{\left\{i_{1}, i_{2}\right\}}=A_{i_{1}} \times A_{i_{2}}$. При этом отображение $\zeta_{\left\{i_{1}, i_{2}\right\}}$ осуществляет изоморфизм решетки $\mathbf{L}_{\left\{i_{1}, i_{2}\right\}}$ и решетки $\left[\pi_{\left\{i_{1}, i_{2}\right\}}^{n}, \nabla_{A}\right]_{\mathbf{L}_{A}}$.

Через $\mathbf{L}_{\left\{i_{1}, i_{2}\right\}}^{*}$ обозначим множество $\mathbf{L}_{\left\{i_{1}, i_{2}\right\}} \backslash \mathbf{D}^{(2)}\left(A_{\left\{i_{1}, i_{2}\right\}}\right)$.

Лемма 4.1. Пусть $\theta \in \mathbf{L}_{\left\{i_{1}, i_{2}\right\}}^{*}$. Тогда отношение $\varphi=\zeta_{\left\{i_{1}, i_{2}\right\}}(\theta)$ является коатомом решетки $\mathbf{L}_{B^{n}}$.

Доказательство. Из формулы (1.5) следует, что $\zeta_{\left\{i_{1}, i_{2}\right\}}\left(\nabla_{A_{\left\{i_{1}, i_{2}\right\}}}\right)=\nabla_{A}$. Поэтому $\varphi \neq \nabla_{A}$.

Таким образом, если утверждение не верно, то существует такое отношение $\rho \in \mathbf{L}_{A}$, что $\theta \subset \rho \subset \nabla_{A}$. Все три отношения, входящие в эту цепочку, принадлежат решетке $\left\lceil\pi_{\left\{i_{1}, i_{2}\right\}}^{n}\right\rceil$. Поэтому определен элемент $\zeta_{\left\{i_{1}, i_{2}\right\}}^{-1}(\rho)$ и имеет место цепочка вложений

$$
\Delta_{A_{\left\{i_{1}, i_{2}\right\}}} \subset \theta \subset \zeta_{\left\{i_{1}, i_{2}\right\}}^{-1}(\rho) \subset \nabla_{\left.A_{\left\{i_{1}, i_{2}\right\}}\right\}},
$$

что противоречит следствию 1.2 .

В дальнейших рассмотрениях нам будет удобно использовать терминологию теории графов. Для этого будем рассматривать каждую пару $\langle V, \Gamma\rangle$, где $V \subseteq Z_{n}, \Gamma \subseteq V^{\{2\}}$, как граф с множеством $V$ вершин и множеством $\Gamma$ ребер, причем ребро $\left\{v_{1}, v_{2}\right\}$ инцидентно вершинам $v_{1}, v_{2}$. 
Будем говорить, что ребра графа $\langle V, \Gamma\rangle$ помечены, если каждому ребру $\left\{v_{1}, v_{2}\right\} \in \Gamma$ сопоставлено отношение $\omega_{\left\{i_{1}, i_{2}\right\}} \in \mathbf{L}_{\left\{i_{1}, i_{2}\right\}}^{*}$. Это эквивалентно тому, что выбран вектор пометок $\left(\omega_{\left\{i_{1}, i_{2}\right\}}\right)_{\left\{i_{1}, i_{2}\right\} \in \Gamma}$, принадлежащий множеству

$$
\Omega\langle V, \Gamma\rangle=\prod_{\left\{i_{1}, i_{2}\right\} \in \Gamma} \mathbf{L}_{\left\{i_{1}, i_{2}\right\}}^{*} .
$$

Отметим, что граф $\langle V, \Gamma\rangle$ нельзя пометить, если для какого-либо ребра $\left\{i_{1}, i_{2}\right\} \in \Gamma$ множество $\mathbf{L}_{\left\{i_{1}, i_{2}\right\}}^{*}$ пусто. Это приводит нас к следующему определению.

Граф $\langle V, \Gamma\rangle$ назовем допустимылм, если

$$
\Gamma \subseteq \Gamma_{\mathbf{L}_{A}} \stackrel{\text { def }}{=}\left\langle\left\{i_{1}, i_{2}\right\} \in Z_{n}^{\{2\}} \mid \mathbf{L}_{\left\{i_{1}, i_{2}\right\}}^{*} \neq \varnothing\right\rangle .
$$

Ясно, что граф можно пометить тогда и только тогда, когда он допустим.

Для вектора пометок

$$
\mathbf{w}=\left(\omega_{\left\{i_{1}, i_{2}\right\}}\right)_{\left\{i_{1}, i_{2}\right\} \in \Gamma}
$$

полагаем

$$
\mathrm{T}(\mathbf{w})=\pi_{Z_{n} \backslash V}^{(n)} \wedge\left(\underset{\left\{i_{1}, i_{2}\right\} \in \Gamma}{\wedge} \zeta_{\left\{i_{1}, i_{2}\right\}}\left(\omega_{\left\{i_{1}, i_{2}\right\}}\right)\right) .
$$

Если $\Gamma^{\prime} \subset \Gamma$, то существует естественное отображение

$$
\Omega\langle S, \Gamma\rangle \rightarrow \Omega\left\langle S, \Gamma^{\prime}\right\rangle
$$

сопоставляющее вектору $\mathbf{w} \in \Omega\langle S, \Gamma\rangle$ вида (4.1) вектор

$$
\left.\mathbf{w}\right|_{\Gamma^{\prime}}=\left(\omega_{\left\{i_{1}, i_{2}\right\}}\right)_{\left\{i_{1}, i_{2}\right\} \in \Gamma^{\prime}} \text {. }
$$

Согласно определениям

$$
\begin{aligned}
\mathrm{T}(\mathbf{w}) & =\pi_{Z_{n} \backslash S}^{(n)} \wedge\left(\underset{\left\{i_{1}, i_{2}\right\} \in \Gamma \backslash \Gamma^{\prime}}{\wedge} \zeta_{\left\{i_{1}, i_{2}\right\}}\left(\omega_{\left\{i_{1}, i_{2}\right\}}\right)\right) \wedge\left({\hat{\left\{i_{1}, i_{2}\right\} \in \Gamma^{\prime}}}_{\left\{i_{1}, i_{2}\right\}}\left(\omega_{\left\{i_{1}, i_{2}\right\}}\right)\right)= \\
& =\mathrm{T}\left(\left.\mathbf{w}\right|_{\Gamma^{\prime}}\right) \wedge(\underbrace{}_{\left\{i_{1}, i_{2}\right\} \in \Gamma \backslash \Gamma^{\prime}} \zeta_{\left\{i_{1}, i_{2}\right\}}\left(\omega_{\left\{i_{1}, i_{2}\right\}}\right))
\end{aligned}
$$

откуда следует включение

$$
\mathrm{T}(\mathbf{w}) \subseteq \mathrm{T}\left(\left.\mathbf{w}\right|_{\Gamma^{\prime}}\right),
$$

выполняющееся для любого вектора $\mathbf{w} \in \Omega\langle S, \Gamma\rangle$. 
В ряде случаев неравенство (4.3) может быть уточнено.

Лемма 4.2. Пусть $d=\left\{u_{1}, u_{2}\right\}$ - такое ребро допустимого графа $\langle V, \Gamma\rangle$, что хотя бы одна из вершин $u_{1}, u_{2}$ является висячей. Тогда для любого вектора $\mathbf{w} \in \Omega\langle V, \Gamma\rangle$ имеет место включение

$$
\mathrm{T}(\mathbf{w}) \triangleleft_{\mathbf{L}_{A}} \mathrm{~T}\left(\left.\mathbf{w}\right|_{\Gamma \backslash\{d\}}\right) .
$$

Доказательство. Для упрощения обозначений будем считать, что $d=\{1,2\}$ и вершина 1 является висячей.

Пусть вектор $\mathbf{w}$ имеет вид (4.1). В рассматриваемом случае равенство (4.2) приобретает вид

$$
\mathrm{T}(\mathbf{w})=\mathrm{T}\left(\left.\mathbf{w}\right|_{\Gamma \backslash\{d\}}\right) \wedge \omega_{\{1,2\}} .
$$

Согласно леммам 1.3, 1.4 и следствию 1.1 либо имеет место включение (4.4), либо имеет место равенство $\mathrm{T}(\mathbf{w})=\mathrm{T}\left(\left.\mathbf{w}\right|_{\Gamma \backslash\{d\}}\right)$, и нам нужно лишь доказать, что последнее равенство невозможно.

Рассмотрим элементы

$$
\mathbf{a}=\left(a_{1}, a_{2}, \ldots, a_{n}\right), \quad \mathbf{b}=\left(b, a_{2}, \ldots, a_{n}\right)
$$

где $a_{i} \in A_{i}-$ произвольные элементы, $b \neq a_{1}$. Так как $1 \notin V$, то $(\mathbf{a}, \mathbf{b}) \in$ $\pi_{Z_{n} \backslash V}^{(n)}$. Пусть $\{i, j\} \in \Gamma \backslash\{d\}$. Так как $1-$ висячая вершина, то $d-$ единственное ребро, ее содержащее. Поэтому $1 \notin\{i, j\}$ и $(\mathbf{a}, \mathbf{b}) \in \pi_{\{i, j\}}^{(n)}$. Отсюда и из включения $\pi_{\{i, j\}}^{(n)} \subset \zeta_{\{i, j\}}\left(\omega_{\{i, j\}}\right)$ следует, что $(\mathbf{a}, \mathbf{b}) \in \zeta_{\{i, j\}}\left(\omega_{\{i, j\}}\right)$. Таким образом,

$$
(\mathbf{a}, \mathbf{b}) \in \mathrm{T}\left(\left.\mathbf{w}\right|_{\Gamma \backslash\{d\}}\right) .
$$

Рассмотрим отношение $\omega_{\{1,2\}} \in \Omega_{\{1,2\}}$. Из теоремы 2.1 следует, что множества $A_{1}, A_{2}$ равномощны. Применив лемму 2.1 , получаем, что существуют биекция $f: A_{1} \rightarrow A_{2}$ и операция $\odot \in \mathcal{Q}\left(A_{2}\right)$, для которых

$$
\left(\left(\left(a_{1}, a_{2}\right),\left(b_{1}, b_{2}\right)\right) \in \omega_{\{1,2\}}\right) \Leftrightarrow\left(f\left(a_{1}\right) \odot a_{2}=f\left(b_{1}\right) \odot b_{2}\right) .
$$

Из свойств квазигрупповых операций следует, что $\left(\left(a_{1}, a_{2}\right),\left(b, a_{2}\right)\right) \notin \omega_{\{1,2\}}$ для всех таких элементов $a_{1}, b \in A_{1}, a_{2} \in A_{2}$, что $b \neq a_{1}$. Поэтому $(\mathbf{a}, \mathbf{b}) \notin$ $\zeta_{\{1,2\}}\left(\omega_{\{1,2\}}\right)$ и $(\mathbf{a}, \mathbf{b}) \notin \mathrm{T}(\mathbf{w})$. Следовательно, $\mathrm{T}(\mathbf{w}) \neq \mathrm{T}\left(\left.\mathbf{w}\right|_{\Gamma \backslash\{d\}}\right)$.

Лемма доказана. 
Лемма 4.3. Пусть $\langle V, \Gamma\rangle$ - ациклический допустимый граф. Тогда для любого вектора $\mathbf{w} \in \Omega\langle V, \Gamma\rangle$ в решетке $\mathbf{L}_{B^{n}}$ существует иель

$$
\mathrm{T}(\mathbf{w})=\tau_{n-|V|+|\Gamma|} \triangleleft_{\mathbf{L}_{A}} \tau_{n-|V|+|\Gamma|-1} \triangleleft_{\mathbf{L}_{A}} \cdots \triangleleft_{\mathbf{L}_{A}} \tau_{1} \triangleleft_{\mathbf{L}_{A}} \tau_{0}=\nabla_{A} .
$$

Доказательство. Будем вести доказательство индукцией по величине $|\Gamma|$.

Если $|\Gamma|=0$, то $\Gamma=\varnothing$ и $\Omega\langle V, \Gamma\rangle$ содержит единственный вектор $\Lambda$ размерности 0. Для этого вектора $\mathrm{T}(\Lambda)=\pi_{Z_{n} \backslash V}^{(n)}$, и утверждение вытекает из следствия 1.2.

Допустим, что утверждение верно для графов, содержащих $k \geqslant 0$ ребер, и рассмотрим ациклический граф $\langle V, \Gamma\rangle$, содержащий $k+1$ ребер. Так как граф $\langle V, \Gamma\rangle$ ацикличен, то его компоненты связности являются деревьями. При сделанном предположении хотя бы одно из них не одноэлементно и, следовательно, содержит висячие вершины (см. [13, гл.4]). Пусть $v$ - одна из висячих вершин и $d \in \Gamma-$ единственное ребро, инцидентное вершине $v$.

Очевидно, что граф $\langle V, \Gamma \backslash\{d\}\rangle$ ацикличен и содержит $k$ ребер. Поэтому применимо предположение индукции и существует цепь

$$
\mathrm{T}\left(\mathbf{w}^{\prime}\right)=\tau_{n-|V|+|\Gamma|-1} \triangleleft_{\mathbf{L}_{A}} \cdots \triangleleft_{\mathbf{L}_{A}} \tau_{1} \triangleleft_{\mathbf{L}_{A}} \tau_{0}=\nabla_{A} .
$$

Объединив эту цепь с включением (4.4), получаем нужную нам цепь.

Теорема 4.1. Следуюшие свойства элемента $\tau$ GA-решетки $\mathbf{L}_{A}$ эквивалентния:

1. Существуют такое допустимое дерево $\langle W, \Gamma\rangle$ и такой вектор пометок $\mathbf{w} \in \Omega\langle W, \Gamma\rangle$, что $\tau=\mathrm{T}(\mathbf{w})$.

2. Отношение $\tau$ является атомом решетки $\mathbf{L}_{A}$.

3. Отномение $\tau$ не равно отношению $\Delta_{A}$ и существуют такой полный допустимый граф $\left\langle V, V^{\{2\}}\right\rangle$ и такой вектор пометок $\mathbf{v} \in \Omega\left\langle V, V^{\{2\}}\right\rangle$, что $\tau \subseteq \mathrm{T}(\mathbf{v})$.

Доказательство. $1 \Rightarrow 2$. Предположим, что $\tau=\mathrm{T}(\mathbf{w})$ для дерева $\langle W, \Gamma\rangle$ и вектора $\mathbf{w} \in \Omega\langle W, \Gamma\rangle$.

По свойству деревьев граф $\langle W, \Gamma\rangle$ ацикличен и содержит $|W|-1$ ребер. Применив лемму 4.3, получаем, что существует цепь

$$
\tau=\tau_{n-1} \triangleleft_{\mathbf{L}_{A}} \tau_{n-2} \triangleleft_{\mathbf{L}_{A}} \cdots \triangleleft_{\mathbf{L}_{A}} \tau_{1} \triangleleft_{\mathbf{L}_{A}} \tau_{0}=\nabla_{A} .
$$


Допустим, что отношение $\tau$ не является атомом. Тогда либо $\tau=\Delta_{A}$, либо существует такой элемент $\tau^{\prime} \in \mathbf{L}_{A}$, что $\tau \supset \tau^{\prime} \supset \Delta_{A}$. В первом случае цепь (4.6) является максимальной цепью решетки $\mathbf{L}_{A}$ и длина этой решетки равна $n-1$. Во втором существует цепь

$$
\nabla_{A}=\tau_{0} \supset \tau_{1} \supset \ldots \supset \tau_{n-1}=\tau \supset \tau^{\prime} \supset \Delta_{A}
$$

и длина решетки $\mathbf{L}_{A}$ больше или равна $n+1$. Оба случая противоречат следствию 1.2. Таким образом, отношение $\tau$ является атомом.

$2 \Rightarrow 3$. Предположим, что отношение $\tau$ является атомом. Положим

$$
V=\left\langle i \in Z_{n} \mid \tau \nsubseteq \pi_{i}^{(n)}\right\rangle .
$$

Из определений и равенства (1.3) следует неравенство

$$
\tau \subseteq \pi_{Z_{n} \backslash V}^{(n)}
$$

Пусть $\left\{i_{1}, i_{2}\right\} \in V^{\{2\}}$. Рассмотрим элемент $\tau^{\prime}=\pi_{\left\{i_{1}, i_{2}\right\}}^{(n)} \vee \tau$. Заметим, что

$$
\tau^{\prime} \notin\left\{\pi_{\left\{i_{1}, i_{2}\right\}}^{(n)}, \pi_{\left\{i_{1}\right\}}^{(n)}, \pi_{\left\{i_{2}\right\}}^{(n)}, \nabla_{A}\right\} .
$$

Действительно, если $\tau^{\prime}=\pi_{\left\{i_{1}, i_{2}\right\}}^{(n)}$ или $\tau^{\prime}=\pi_{\left\{i_{j}\right\}}^{(n)}$, то $\tau \subseteq \pi_{i_{j}}^{(n)}$, что противоречит определению множества $V$. Далее, если $\tau^{\prime}=\nabla_{A}$, то из леммы 1.4 и следствия 1.1 вытекают включения

$$
\pi_{\left\{i_{1}, i_{2}\right\}}^{(n)} \triangleleft_{\mathbf{L}_{A}} \pi_{\left\{i_{1}\right\}}^{(n)} \triangleleft_{\mathbf{L}_{A}} \nabla_{A}, \quad \pi_{\left\{i_{1}, i_{2}\right\}}^{(n)} \triangleleft_{\mathbf{L}_{A}} \nabla_{A},
$$

что невозможно для модулярных решеток. Теперь имеем

$$
\begin{gathered}
\zeta_{\left\{i_{1}, i_{2}\right\}}^{-1}\left(\tau^{\prime}\right) \in \mathbf{L}_{\left\{i_{1}, i_{2}\right\}} \backslash \zeta_{\left\{i_{1}, i_{2}\right\}}^{-1}\left(\left\{\pi_{\left\{i_{1}, i_{2}\right\}}^{(n)}, \pi_{\left\{i_{1}\right\}}^{(n)}, \pi_{\left\{i_{2}\right\}}^{(n)}, \nabla_{A}\right\}\right)= \\
=\mathbf{L}_{\left\{i_{1}, i_{2}\right\}} \backslash \mathbf{D}^{(2)}\left(A_{\left\{i_{1}, i_{2}\right\}}\right)=\mathbf{L}_{\left\{i_{1}, i_{2}\right\}}^{*} .
\end{gathered}
$$

Последнее включение означает, что $\mathbf{L}_{\left\{i_{1}, i_{2}\right\}}^{*} \neq \varnothing$. Так как ребро $\left\{i_{1}, i_{2}\right\} \in$ $V^{\{2\}}$ выбиралось произвольно, то граф $\left\langle V, V^{\{2\}}\right\rangle$ допустим.

Элемент $\zeta_{\left\{i_{1}, i_{2}\right\}}^{-1}\left(\tau^{\prime}\right)$ обозначим через $\varphi_{\left\{i_{1}, i_{2}\right\}}$. Имеем

$$
\tau \subseteq \pi_{\left\{i_{1}, i_{2}\right\}}^{(n)} \vee \tau \subseteq \zeta_{\left\{i_{1}, i_{2}\right\}}\left(\varphi_{\left\{i_{1}, i_{2}\right\}}\right) .
$$

Положим $\mathbf{v}=\left(\varphi_{\left\{i_{1}, i_{2}\right\}}\right)_{\left\{i_{1}, i_{2}\right\} \in V\{2\}}$. Из неравенств (4.7) и (4.8) следует неравенство $\tau \subseteq \mathrm{T}(\mathbf{v})$, и условие 3 выполняется. 
$3 \Rightarrow 1$. Пусть $\Delta_{A} \subset \tau \subseteq \mathrm{T}(\mathbf{v})$ для вектора пометок $\mathbf{v} \in \Omega\left\langle V, V^{\langle 2\rangle}\right\rangle$. Выберем произвольное дерево $\langle V, \Gamma\rangle$ с множеством $V$ вершин. Непосредственно из определений следует, что каждый подграф допустимого графа сам является допустимым. Поэтому дерево $\langle V, \Gamma\rangle$ допустимо. Из неравенства (4.3) следует неравенство

$$
\Delta_{A} \subset \tau \subseteq \mathrm{T}(\mathbf{v}) \subseteq \mathrm{T}\left(\left.\mathbf{v}\right|_{\Gamma}\right) .
$$

Согласно доказанной нами импликации $1 \Rightarrow 2$ отношение $\mathrm{T}\left(\left.\mathbf{v}\right|_{\Gamma}\right)$ является атомом решетки $\mathbf{L}_{A}$. Из определения атомов и предыдущих рассуждений следует, что $\tau=\mathrm{T}\left(\left.\mathbf{v}\right|_{\Gamma}\right)$.

Импликация $1 \Rightarrow 3$ означает, что для любого допустимого дерева $\langle W, \Gamma\rangle$ и любого вектора пометок $\mathbf{w} \in \Omega\langle W, \Gamma\rangle$ найдутся такой допустимый полный граф $\left\langle V, V^{\langle 2\rangle}\right\rangle$ и такой вектор пометок $\mathbf{v} \in \Omega\left\langle V, V^{\langle 2\rangle}\right\rangle$, что $\mathrm{T}(\mathbf{w}) \subseteq \mathrm{T}(\mathbf{v})$. Нам необходимо усилить это утверждение.

Теорема 4.2. Пусть $\langle W, \Gamma\rangle-$ допустимое дерево. Тогда полный граф $\left\langle W, W^{\{2\}}\right\rangle$ допустим и для каждого вектора $\mathbf{w} \in \Omega\langle W, \Gamma\rangle$ существует такой вектор $\mathbf{v} \in \Omega\left\langle W, W^{\{2\}}\right\rangle$, что $\mathrm{T}(\mathbf{w})=\mathrm{T}(\mathbf{v}) u \mathbf{w}=\left.\mathbf{v}\right|_{\Gamma}$.

Доказательство. Если $|W| \leqslant 2$, то дерево $\langle W, \Gamma\rangle$ является полным графом, и утверждение очевидно.

Пусть $|W|=3$. Для упрощения обозначений положим $W=\{1,2,3\}$, $\Gamma=\{\{1,2\},\{1,3\}\}$. Пусть $\mathbf{w}=\left(\omega_{\{1,2\}}, \omega_{\{2,3\}}\right)$ - вектор пометок графа $\langle W, \Gamma\rangle$.

В силу теоремы 4.1 отношение $\tau=\mathrm{T}\left(\left(\omega_{\{1,2\}}, \omega_{\{2,3\}}\right)\right)$ является атомом. Из той же теоремы следует, что $\tau \subseteq \mathrm{T}(\mathbf{v})$ для подходящего вектора пометок $\mathbf{v}=\left(\varphi_{\{i, j\}}\right)_{\{i, j\} \in V\{2\}}$ ребер полного допустимого графа $\left\langle V, V^{\langle 2\rangle}\right\rangle$.

Справедливы формула (4.5) и аналогичная формула для отношения $\omega_{\{2,3\}}$ :

$$
\begin{aligned}
& \left(\left(\left(a_{1}, a_{2}\right),\left(b_{1}, b_{2}\right)\right) \in \omega_{\{1,2\}}\right) \Leftrightarrow\left(f\left(a_{1}\right) \odot_{12} a_{2}=f\left(b_{1}\right) \odot_{12} b_{2}\right), \\
& \left(\left(\left(a_{1}, a_{2}\right),\left(b_{1}, b_{2}\right)\right) \in \omega_{\{2,3\}}\right) \Leftrightarrow\left(g\left(a_{1}\right) \odot_{23} a_{2}=g\left(b_{1}\right) \odot_{23} b_{2}\right),
\end{aligned}
$$

где $f: A_{1} \rightarrow A_{2}, g: A_{2} \rightarrow A_{3}$ - биекции, $\odot_{12}, \odot_{23}$ - квазигрупповые операции на множествах $A_{2}$ и $A_{3}$ соответственно.

Выберем произвольно вектор $\mathbf{a}=\left(a_{1}, \ldots, a_{n}\right) \in A$ и элемент $b_{1} \in$ $A_{1} \backslash\left\{a_{1}\right\}$. В качестве $b_{2}$ и $b_{3}$ возьмем решения уравнений

$$
f\left(a_{1}\right) \odot_{12} a_{2}=f\left(b_{1}\right) \odot_{12} x, \quad g\left(a_{2}\right) \odot_{23} a_{3}=g\left(b_{2}\right) \odot_{23} y .
$$


Теперь из определений следует, что

$$
\begin{aligned}
& \left(\left(a_{1}, a_{2}, a_{3}, a_{4}, \ldots, a_{n}\right),\left(b_{1}, b_{2}, b_{3}, a_{4}, \ldots, a_{n}\right)\right) \in \tau, \\
& \left(\left(a_{1}, a_{2}, a_{3}, a_{4}, \ldots, a_{n}\right),\left(b_{1}, b_{2}, b_{3}, a_{4}, \ldots, a_{n}\right)\right) \notin \pi_{1}^{(n)}
\end{aligned}
$$

и $\tau \nsubseteq \pi_{1}^{(n)}$. Следовательно, $\mathrm{T}(\mathbf{v}) \nsubseteq \pi_{1}^{(n)}$, что возможно только в случае $1 \in V$.

Так же показываем, что $3 \in V$.

Поскольку ребра $\{1,2\},\{2,3\},\{1,3\}$ входят в допустимые графы, постольку они принадлежат множеству $\Gamma_{\mathbf{L}_{A}}$ и полный граф $\langle\{1,2,3\},\{\{1,2\},\{2,3\},\{1,3\}\}\rangle$ допустим. Имеем $\tau \subseteq \mathrm{T}(\mathbf{v}) \subseteq \zeta_{\{1,3\}}\left(\varphi_{\{1,3\}}\right)$,

$$
\begin{aligned}
\tau=\tau \wedge \zeta_{\{1,3\}}\left(\varphi_{\{1,3\}}\right) & =\zeta_{\{1,2\}}\left(\omega_{\{1,2\}}\right) \wedge \zeta_{\{2,3\}}\left(\omega_{\{2,3\}}\right) \wedge \zeta_{\{1,3\}}\left(\varphi_{\{1,3\}}\right)= \\
& =\mathrm{T}\left(\left(\omega_{\{1,2\}}, \omega_{\{2,3\}}, \varphi_{\{1,3\}}\right)\right),
\end{aligned}
$$

и утверждение верно.

Пусть теперь $|W| \geqslant 4$. Будем говорить, что вектор пометок $\mathbf{w}^{\prime} \in \Omega\left(W, \Gamma^{\prime}\right)$, где $\Gamma \subseteq \Gamma^{\prime} \subseteq W^{\{2\}}$, является продолжением вектора $\mathbf{w} \in \Omega(W, \Gamma)$, если $\mathrm{T}(\mathbf{w})=\mathrm{T}\left(\mathbf{w}^{\prime}\right)$ и $\mathbf{w}=\left.\mathbf{w}^{\prime}\right|_{\Gamma}$.

Пусть $\mathfrak{G}-$ множество таких подмножеств $\Gamma^{\prime}$ множества $W^{\{2\}}$, что $\Gamma \subseteq \Gamma^{\prime}$ и для каждого вектора $\mathbf{w} \in \Omega(W, \Gamma)$ существует продолжение $\mathbf{w}^{\prime} \in \Omega\left(W, \Gamma^{\prime}\right)$.

Ясно, что $\Gamma \in \mathfrak{G}$. Поэтому множество $\mathfrak{G}$ не пусто. Так как множество $W^{\{2\}}$ конечно, то конечным будет и множество $\mathfrak{G}$. Поэтому оно содержит максимальные относительно теоретико-множественного порядка элементы. Пусть $\Gamma^{(0)}-$ один из таких элементов. Предположим, что $\Gamma^{(0)} \neq W^{\{2\}}$.

Так как $\Gamma \subseteq \Gamma^{(0)}$ и граф $\langle W, \Gamma\rangle$ связен, то связен и граф $\left\langle W, \Gamma^{(0)}\right\rangle$. Поэтому при сделанном предположении существуют такие ребра $\left\{i_{1}, i_{2}\right\},\left\{i_{2}, i_{3}\right\} \in \Gamma^{(0)}$, что $i_{1} \neq i_{3}$ и $\left\{i_{1}, i_{3}\right\} \notin \Gamma^{(0)}$.

Рассмотрим произвольный вектор пометок $\mathbf{w}=\left(\omega_{\{i, j\}}\right)_{\{i, j\} \in \Gamma}$ и его продолжение $\mathbf{w}^{(0)}=\left(\omega_{\{i, j\}}\right)_{\{i, j\} \in \Gamma^{(0)}}$. Также рассмотрим граф $\left\langle\left\{i_{1}, i_{2}, i_{3},\left\{\left\{i_{1}, i_{2}\right\},\left\{i_{2}, i_{3}\right\}\right\}\right\rangle\right.$. Тогда пара $\left(\omega_{\left\{i_{1}, i_{2}\right\}}, \omega_{\left\{i_{2}, i_{3}\right\}}\right)$ является вектором пометок для него. Так как число вершин этого графа равно 3, то доказываемое утверждение для него верно. Это означает, что $\left\{i_{1}, i_{3}\right\} \in \Gamma_{\mathbf{L}_{A}}$ и существует такое отношение $\omega_{\left\{i_{1}, i_{3}\right\}}$, что

$$
\begin{aligned}
& \pi_{Z_{n} \backslash\left\{i_{1}, i_{2}, i_{3}\right\}}^{(n)} \wedge \zeta_{\left\{i_{1}, i_{2}\right\}}\left(\omega_{\left\{i_{1}, i_{2}\right\}}\right) \wedge \zeta_{\left\{i_{2}, i_{3}\right\}}\left(\omega_{\left\{i_{2}, i_{3}\right\}}\right)= \\
& =\pi_{Z_{n} \backslash\left\{i_{1}, i_{2}, i_{3}\right\}}^{(n)} \wedge \zeta_{\left\{i_{1}, i_{2}\right\}}\left(\omega_{\left\{i_{1}, i_{2}\right\}}\right) \wedge \zeta_{\left\{i_{2}, i_{3}\right\}}\left(\omega_{\left\{i_{2}, i_{3}\right\}}\right) \wedge \zeta_{\left\{i_{2}, i_{2}\right\}}\left(\omega_{\left\{i_{1}, i_{3}\right\}}\right) .
\end{aligned}
$$


Отсюда и из леммы 1.4 следует равенство

$$
\begin{aligned}
& \zeta_{\left\{i_{1}, i_{2}\right\}}\left(\omega_{\left\{i_{1}, i_{2}\right\}}\right) \wedge \zeta_{\left\{i_{2}, i_{3}\right\}}\left(\omega_{\left\{i_{1}, i_{3}\right\}}\right)= \\
& =\zeta_{\left\{i_{1}, i_{2}\right\}}\left(\omega_{\left\{i_{1}, i_{2}\right\}}\right) \wedge \zeta_{\left\{i_{2}, i_{3}\right\}}\left(\omega_{\left\{i_{2}, i_{3}\right\}}\right) \wedge \zeta_{\left\{i_{1}, i_{2}\right\}}\left(\omega_{\left\{i_{1}, i_{3}\right\}}\right),
\end{aligned}
$$

и мы имеем

$$
\begin{aligned}
& \pi_{Z_{n} \backslash W}^{(n)} \wedge\left(\bigwedge_{\{i, j\} \in \Gamma^{(0)}} \zeta_{\{i, j\}}\left(\omega_{\{i, j\}}\right)\right) \wedge \zeta_{\left\{i_{1}, i_{3}\right\}}\left(\omega_{\left\{i_{1}, i_{3}\right\}}\right)= \\
& =\pi_{Z_{n} \backslash W}^{(n)} \wedge\left(\bigwedge_{\{i, j\} \in \Gamma^{(0)}} \zeta_{\{i, j\}}\left(\omega_{\{i, j\}}\right)\right)=\pi_{Z_{n} \backslash W}^{(n)} \wedge\left(\bigwedge_{\{i, j\} \in \Gamma^{(0)}} \zeta_{\{i, j\}}\left(\omega_{\{i, j\}}\right)\right) .
\end{aligned}
$$

Таким образом, любой вектор пометок продолжается до вектора пометок, принадлежащего множеству $\Omega\left\langle W, \Gamma^{(0)} \cup\left\{\left\{i_{1}, i_{3}\right\}\right\}\right\rangle$, что противоречит предположению о максимальности множества $\Gamma^{(0)}$.

Полученное противоречие означает, что $\Gamma^{(0)}=W^{\{2\}}$, поэтому утверждение теоремы верно.

Следствие 4.1. Каждая компонента связности графа $\left\langle Z_{n}, \Gamma_{\mathbf{L}_{A}}\right\rangle$ является полным графом.

Утверждение очевидным образом вытекает из теоремы 4.2.

Теорема 4.3. Каждый элемент $G A$-решетки $\mathbf{L}_{A}$ разлагается в объединение некоторого множества ее атомов.

Доказательство. Пусть $\theta \in \mathbf{L}_{A}$. Если $\ell(\theta)=0$, то $\theta=\Delta_{A}$ и $\theta-$ объединение пустого множества атомов. Если $\ell(\theta)=1$, то $\theta$ является атомом и утверждение верно.

Допустим, что $\ell(\theta)=m+1 \geqslant 2$ и утверждение верно для всех элементов меньшей высоты. Так как решетка $\mathbf{L}_{A}$ конечна, то существует такой атом $\tau$ решетки $\mathbf{L}_{A}$, что $\tau \subset \theta$.

Из равенств (1.2), (1.3) следует соотношение $\tau \supset \Delta_{A}=\wedge_{k=1}^{n} \pi_{k}^{(n)}$. Поэтому найдется такой номер $k$, что $\tau \nsubseteq \pi_{k}^{(n)}$.

Полагаем $\theta^{\prime}=\theta \wedge \pi_{k}^{(n)}$. Если $\theta^{\prime} \subseteq \theta$, то $\pi_{k}^{(n)} \supseteq \theta \wedge \pi_{k}^{(n)}=\theta^{\prime}=\theta \supseteq \tau$, что противоречит выбору номера $k$. Значит, $\theta^{\prime} \subset \theta$, откуда следует неравенство $\ell\left(\theta^{\prime}\right)<\ell(\theta)$. По предположению индукции элемент $\theta^{\prime}$ разлагается в объединение атомов $\theta^{\prime}=\vee_{j=1}^{r} \tau_{j}$.

Из тождества модулярности следует

$$
\theta^{\prime} \vee \tau=\theta \wedge\left(\pi_{k}^{(n)} \vee \tau\right)=\theta \wedge \nabla_{A}=\theta, \quad \theta=\theta^{\prime} \vee \tau=\left(\bigvee_{j=1}^{r} \tau_{j}\right) \vee \tau,
$$

что дает интересующее нас разложение. 
Теорема 4.4. GA-решетка $\mathbf{L}_{A}$ неразложима тогда и только тогда, когда граф $\left\langle Z_{n}, \Gamma_{\mathbf{L}_{A}}\right\rangle$ является полным графом.

Доказательство. Предположим, что решетка $\mathbf{L}_{A}$ разложима и отображение $\zeta_{I} \wedge \zeta_{Z_{n} \backslash I}$ является изоморфизмом. Допустим, что существуют такие элементы $i_{1} \in I, i_{2} \in Z_{n} \backslash I$, что $\left\{i_{1}, i_{2}\right\} \in \Gamma_{\mathbf{L}_{A}}$. Последнее означает, что множество $\mathbf{L}_{\left\{i_{1}, i_{2}\right\}}^{*}$ не пусто. Пусть $\omega-$ элемент из множества $\mathbf{L}_{\left\{i_{1}, i_{2}\right\}}^{*}$. Тогда $G=\left\langle\left\{i_{1}, i_{2}\right\},\left\{\left\{i_{1}, i_{2}\right\}\right\}\right\rangle$ - допустимый граф и $(\omega) \in \Omega(G)$. Это позволяет построить атом $\tau=\mathrm{T}(\omega) \in \mathbf{L}_{A}$.

Для отношения $\omega$ справедлив аналог формулы (4.5). Используя эту формулу, аналогично доказательству леммы 4.2 показываем, что $\tau \nsubseteq \pi_{I}^{(n)}, \tau \nsubseteq$ $\pi_{Z_{n} \backslash I}^{(n)}$. Так как отношение $\tau$ является атомом, то

$$
\Delta_{A}=\tau \wedge \pi_{I}^{(n)}=\tau \wedge \pi_{Z_{n} \backslash I}^{(n)} .
$$

Из сделанного предположения следует, что

$$
\mathrm{T}(\omega)=\zeta_{I}\left(\theta_{1}\right) \wedge \zeta_{Z_{n} \backslash I}\left(\theta_{2}\right)
$$

для некоторых отношений $\theta_{1} \in \mathbf{L}_{A^{I}}, \theta_{2} \in \mathbf{L}_{A^{z_{n} \backslash I}}$. Используя равенство (4.9), получаем

$\pi_{I}^{(n)} \wedge \pi_{Z_{n} \backslash I}^{(n)}=\Delta_{A}=\pi_{I}^{(n)} \wedge \tau=\pi_{I}^{(n)} \wedge \zeta_{I}\left(\theta_{1}\right) \wedge \zeta_{Z_{n} \backslash I}\left(\theta_{2}\right)=\pi_{I}^{(n)} \wedge \zeta_{Z_{n} \backslash I}\left(\theta_{2}\right)$.

Из леммы 1.4 следует, что $\pi_{Z_{n} \backslash I}^{(n)}=\zeta_{Z_{n} \backslash I}\left(\theta_{2}\right)$ и

$$
\mathrm{T}(\omega)=\zeta_{I}\left(\theta_{1}\right) \wedge \pi_{Z_{n} \backslash I}^{(n)} \subseteq \pi_{Z_{n} \backslash I}^{(n)},
$$

что противоречит неравенствам (4.9).

Полученное противоречие показывает, что таких смежных вершин $i_{1}, i_{2}$, что $i_{1} \in I, i_{2} \in Z_{n} \backslash I$, не существует, и граф $\left\langle Z_{n}, \Gamma_{\mathbf{L}_{A}}\right\rangle$ не связен. Тем более он не является полным.

Теперь предположим, что граф $\left\langle Z_{n}, \Gamma_{\mathbf{L}_{A}}\right\rangle$ не полон. В силу следствия 4.1 он не связен. Пусть $I-$ одна из компонент связности.

Рассмотрим произвольный атом $\tau$ решетки $\mathbf{L}_{A}$. В силу теоремы 4.1 существуют такое допустимое дерево $\langle V, \Gamma\rangle$ и такой вектор $\mathbf{w} \in \Omega\langle V, \Gamma\rangle$, что $\tau=\mathrm{T}(\mathbf{w})$. При сделанном предположении либо $V \subseteq I$, либо $V \subseteq Z_{n} \backslash I$.

Если $V \subseteq I$, то $\tau \subseteq \pi_{Z_{n} \backslash I}^{(n)}$. Отсюда и из тождества модулярности следует, что

$$
\left(\tau \vee \pi_{I}^{(n)}\right) \wedge \pi_{Z_{n} \backslash I}^{(n)}=\tau \vee\left(\pi_{I}^{(n)} \wedge \pi_{Z_{n} \backslash I}^{(n)}\right)=\tau \vee \Delta_{A}=\tau
$$


Так как $\nabla_{A} \supseteq \tau \vee \pi_{I}^{(n)} \supseteq \pi_{I}^{(n)}$, то существует такой элемент $\rho \in \mathbf{L}_{A^{I}}$, что $\tau \vee \pi_{I}^{(n)}=\zeta_{I}(\rho)$. Также имеем $\pi_{Z_{n} \backslash I}^{(n)}=\zeta_{Z_{n} \backslash I}\left(\Delta_{A_{Z_{n} \backslash I}}\right)$. Поэтому равенство (4.10) можно записать в виде

$$
\tau=\zeta_{I}(\rho) \wedge \zeta_{Z_{n} \backslash I}\left(\Delta_{A_{Z_{n} \backslash I}}\right)
$$

T. e.

$$
\tau \in \zeta_{I}\left(\mathbf{L}_{A^{I}}\right) \wedge \zeta_{Z_{n} \backslash I}\left(\mathbf{L}_{A_{Z_{n} \backslash I}}\right)
$$

Так же доказываем, что включение (4.11) выполняется и в случае $V \subseteq Z_{n} \backslash I$

Отсюда и из теоремы 4.3 следует, что $\mathbf{L}_{A}=\zeta_{I}\left(\mathbf{L}_{A^{I}}\right) \wedge \zeta_{Z_{n} \backslash I}\left(\mathbf{L}_{A_{Z_{n}} \backslash I}\right)$, и решетка $\mathbf{L}_{A}$ разложима.

Следствие 4.2. Если $A=\prod_{i=1}^{n} A_{i} u$ GA-решетка $\mathbf{L}_{A}$ неразложима, то все множества $A_{i}$ имеют одинаковую мощность.

Доказательство. Пусть $i, j \in Z_{n}$ и $i<j$. Из теоремы 4.4 следует, что $G A$-решетка $\mathbf{L}_{\{i, j\}}$ отношений эквивалентности на множестве $A_{i} \times A_{j}$ не равна $\mathbf{D}^{(2)}\left(A_{1} \times A_{2}\right)$. Из теоремы 2.1 следует, что множества $A_{i}, A_{j}$ имеют одинаковую мощность.

Так как номера $i, j$ выбирались произвольно, то все множества $A_{i}$ имеют одинаковую мощность.

\section{5. І-системы}

В настоящем разделе проводятся некоторые рассмотрения, которые понадобятся при описании $G A$-решеток больших длин. Так же, как выше, $B-$ конечное множество.

Пусть $\circledast_{1}, \circledast{ }_{2}, \circledast 3 \in \mathcal{Q}(B)$. Определим на множестве $B^{3}$ отношения эквивалентности $\theta_{1}, \theta_{2}, \theta_{3}$, положив

$$
\begin{aligned}
& \left(\left(b_{1}, b_{2}, b_{3}\right),\left(b_{1}^{\prime}, b_{2}^{\prime}, b_{3}^{\prime}\right) \in \theta_{1}\right) \Leftrightarrow\left(b_{2} \circledast_{1} b_{3}=b_{2}^{\prime} \circledast_{1} b_{3}^{\prime}\right), \\
& \left(\left(b_{1}, b_{2}, b_{3}\right),\left(b_{1}^{\prime}, b_{2}^{\prime}, b^{\prime}{ }_{3}\right) \in \theta_{2}\right) \Leftrightarrow\left(b_{1} \circledast_{2} b_{3}=b_{1}^{\prime} \circledast_{2} b_{3}^{\prime}\right) \text {, } \\
& \left(\left(b_{1}, b_{2}, b_{3}\right),\left(b_{1}^{\prime}, b_{2}^{\prime}, b_{3}^{\prime}\right) \in \theta_{3}\right) \Leftrightarrow\left(b_{1} \circledast_{3} b_{2}=b_{1}^{\prime} \circledast_{3} b_{2}^{\prime}\right) \text {. }
\end{aligned}
$$

Скажем, что упорядоченная тройка $(\circledast 1, \circledast 2, \circledast 3)$ инциидентна, если выполняется включение

$$
\theta_{2} \wedge \theta_{3} \subseteq \theta_{1}
$$


Лемма 5.1. Пусть тройка $\left\langle\circledast_{1}, \circledast_{2}, \circledast_{3}\right\rangle$ квазигрупповых операций инцидентна. Тогда операчии $\circledast_{1}, \circledast_{2}, \circledast_{3}$ изотопны некоторой групповой операчии + , определенной на множестве $B$, т.е.

$$
x \circledast{ }_{i} y=\kappa_{i}^{-1}\left(\lambda_{i}(x)+\mu_{i}(y)\right)
$$

для подходящих подстановок $\kappa_{i}, \lambda_{i}, \mu_{i}$, причем подстановки $\lambda_{i}, \mu_{i}$ могут быть выбраны так, что

$$
\lambda_{i}(0)=\mu_{i}(0)=0 .
$$

Доказательство. Пусть $c_{2}, c_{3} \in B$. Выберем элементы $b_{1}, b_{2}, b_{3} \in B$ так, чтобы

$$
c_{2}=b_{1} \circledast_{3} b_{2}, \quad c_{3}=b_{1} \circledast_{2} b_{3} .
$$

Например, это можно сделать следующим образом: элемент $b_{1} \in B$ выбираем произвольно, а в качестве элементов $b_{2}, b_{3} \in B$ берем решения уравнений $c_{2}=b_{1} \circledast_{3} y$ и $c_{3}=b_{1} \circledast_{2} y$ соответственно. Эти уравнения однозначно разрешимы по определению квазигрупп.

Полагаем $c_{2} \odot c_{3}=b_{2} \circledast_{1} b_{3}$. Определение этой операции корректно. Действительно, если $b_{1}^{\prime}, b_{2}^{\prime}, b_{3}^{\prime} \in B$ - другие элементы и выполняются равенства

$$
c_{2}=b_{1}^{\prime} \circledast_{3} b_{2}^{\prime}, \quad c_{3}=b_{1}^{\prime} \circledast_{2} b_{3}^{\prime},
$$

To

$$
b_{1} \circledast_{2} b_{3}=b_{1}^{\prime} \circledast_{2} b_{3}^{\prime}, \quad b_{1} \circledast_{3} b_{2}=b_{2}^{\prime} \circledast_{3} b_{3}^{\prime} .
$$

Из определений (5.1) следует, что справедливо включение

$$
\left(\left(b_{1}, b_{2}, b_{3}\right),\left(b_{1}^{\prime}, b_{2}^{\prime}, b_{3}^{\prime}\right)\right) \in \theta_{2} \wedge \theta_{3} .
$$

Из (5.2) следует, что $\left(\left(b_{1}, b_{2}, b_{3}\right),\left(b^{\prime}{ }_{1}, b_{2}^{\prime}, b_{3}^{\prime}\right)\right) \in \theta_{1}$ и $b_{2}^{\prime} \circledast_{1} b_{3}^{\prime}=b_{2} \circledast_{1} b_{3}=$ $c_{2} \odot c_{3}$.

Таким образом, нами определена операция $\odot$.

Рассмотрим уравнение $c_{2} \odot x=d$. Чтобы решить его, опять выберем разложение $c_{2}=b_{1} \circledast_{3} b_{2}$. Пусть $a$ есть решение уравнения $b_{2} \circledast_{1} x=d$. Тогда

$$
c_{2} \odot\left(b_{1} \circledast a\right)=\left(b_{1} \circledast_{3} b_{2}\right) \odot\left(b_{1} \circledast a\right)=b_{2} \circledast_{1} a=d,
$$

т. е. рассматриваемое уравнение разрешимо. Однозначность решения вытекает из конечности множества $B$. 
Так же показываем, что каждое уравнение $x \odot c_{3}=d$ имеет единственное решение.

Таким образом, операция $\odot$ является квазигрупповой.

Произвольно выберем элементы $b_{1}, b_{2}, b_{3} \in B$. Имеют место тавтологии

$$
b_{1} \circledast_{3} b_{2}=b_{1} \circledast_{3} b_{2}, \quad b_{1} \circledast_{2} b_{3}=b_{1} \circledast_{2} b_{3} .
$$

Теперь из определения операции $\odot$ следует, что

$$
\left(b_{1} \circledast_{3} b_{2}\right) \odot\left(b_{1} \circledast_{2} b_{3}\right)=b_{2} \circledast_{1} b_{3},
$$

т. е. операции $\circledast_{1}, \circledast_{2}, \circledast_{3}, \odot$ связаны общим тождеством транзитивности.

В [2] доказано, что все операции, входящие в общее тождество транзитивности, изотопны некоторой групповой операции.

Возможность выбора подстановок, обладающих свойством (5.4), вытекает из леммы 3.1.

Теорема 5.1. Пусть $\langle B ;+\rangle-$ конечная группа, $\circledast_{1}, \circledast_{2}, \circledast_{3}-$ операции, заданные равенствами (5.3), в которых подстановки $\lambda_{i}, \mu_{i}$ удовлетворяют равенствам (5.4), и тройка $\left\langle\circledast_{1}, \circledast_{2}, \circledast_{3}\right\rangle$ инщидентна. Тогда существуют такие автоморфизмы $h, f$ группы $\langle B ;+\rangle$, что выполняются равенства $\lambda_{3}=\left(f^{-1} h\right) \lambda_{2}, \mu_{3}=-f \lambda_{1}, \mu_{2}=h \mu_{1}$.

Доказательство. Выберем произвольно элементы $u, v \in B$. Для каждого $a \in B$ полагаем

$$
c_{u v}(a)=\left(a, \mu_{3}^{-1}\left(-\lambda_{3}(a)+u\right), \mu_{2}^{-1}\left(-\lambda_{2}(a)+v\right)\right) .
$$

Имеем

$$
\begin{aligned}
& \kappa_{2}^{-1}\left(\lambda_{2}(a)+\mu_{2}\left(\mu_{2}^{-1}\left(-\lambda_{2}(a)+v\right)\right)\right)=\kappa_{2}^{-1}(v), \\
& \kappa_{3}^{-1}\left(\lambda_{3}(a)+\mu_{3}\left(\mu_{3}^{-1}\left(-\lambda_{3}(a)+u\right)\right)\right)=\kappa_{3}^{-1}(u) .
\end{aligned}
$$

Из этих равенств следует, что каждая пара $\left(c_{u v}(a), c_{u v}\left(a^{\prime}\right)\right)$ принадлежит отношению $\theta_{2} \wedge \theta_{3}$. Следовательно, каждая такая пара находится в отношении $\theta_{1}$.

В частности, $\left(c_{u v}(a), c_{u v}(0)\right) \in \theta_{1}$. Это означает, что выполняется равенство

$$
\kappa_{1}^{-1}\left(\lambda_{1} \mu_{3}^{-1}\left(-\lambda_{3}(a)+u\right)+\mu_{1} \mu_{2}^{-1}\left(-\lambda_{2}(a)+v\right)\right)=\kappa_{1}^{-1}\left(\lambda_{1} \mu_{3}^{-1}(u)+\mu_{1} \mu_{2}^{-1}(v)\right) .
$$

Применив к обеим частям равенства подстановку и сгруппировав члены, получим

$$
\mu_{1} \mu_{2}^{-1}\left(-\lambda_{2}(a)+v\right)-\mu_{1} \mu_{2}^{-1}(v)=-\lambda_{1} \mu_{3}^{-1}\left(-\lambda_{3}(a)+u\right)+\lambda_{1} \mu_{3}^{-1}(u) .
$$

Левая часть равенства не зависит от величины $u$, а правая - от величины $v$. 
Поэтому обе части равенства не зависят существенно от этих величин и для некоторой функции $\gamma$

$$
\begin{gathered}
\mu_{1} \mu_{2}^{-1}\left(-\lambda_{2}(a)+v\right)-\mu_{1} \mu_{2}^{-1}(v)=\gamma(a), \\
-\lambda_{1} \mu_{3}^{-1}\left(-\lambda_{3}(a)+u\right)+\lambda_{1} \mu_{3}^{-1}(u)=\gamma(a) .
\end{gathered}
$$

Эти равенства можно записать в эквивалентном виде

$$
\begin{gathered}
\mu_{1} \mu_{2}^{-1}(c+v)=\gamma\left(-\lambda_{2}^{-1}(c)\right)+\mu_{1} \mu_{2}^{-1}(v), \\
-\lambda_{1} \mu_{3}^{-1}(d+u)=\gamma\left(-\lambda_{3}^{-1}(d)\right)-\lambda_{1} \mu_{3}^{-1}(u) .
\end{gathered}
$$

Положив $u=v=0$, получим

$$
\mu_{1} \mu_{2}^{-1}(c)=\gamma\left(-\lambda_{2}^{-1}(c)\right), \quad-\lambda_{1} \mu_{3}^{-1}(d)=\gamma\left(-\lambda_{3}^{-1}(d)\right) .
$$

Подставив последние выражения в равенства (5.5), получим

$$
\begin{aligned}
\mu_{1} \mu_{2}^{-1}(c+v) & =\mu_{1} \mu_{2}^{-1}(c)+\mu_{1} \mu_{2}^{-1}(v), \\
-\lambda_{1} \mu_{3}^{-1}(d+u) & =-\lambda_{1} \mu_{3}^{-1}(d)-\lambda_{1} \mu_{3}^{-1}(u) .
\end{aligned}
$$

Таким образом, отображения $\mu_{1} \mu_{2}^{-1},-\lambda_{1} \mu_{3}^{-1}$ являются автоморфизмами, которые обозначим через $h$ и $f$ соответственно.

Из равенств (5.6) следует, что отображение является подстановкой. Поэтому из них же следуют равенства

$$
\begin{gathered}
\lambda_{2}^{-1}(c)=-\gamma^{-1} h(c), \quad \lambda_{2}(c)=h^{-1} \gamma(-c), \\
\lambda_{3}^{-1}(d)=-\gamma^{-1} f(d), \\
\lambda_{3}(d)=f^{-1} \gamma(-d)=\left(f^{-1} h\right) h^{-1} \gamma(-d)=\left(f^{-1} h\right) \lambda_{2}(d) .
\end{gathered}
$$

Пусть $\Omega_{1}, \Omega_{2}, \Omega_{3} \subseteq \mathcal{Q}(B)$. Скажем, что упорядоченная тройка $\left\langle\Omega_{1}, \Omega_{2}, \Omega_{3}\right\rangle$ образует I-системy, если для любой подстановки $s$ множества $\{1,2,3\}$ и любых операций $\circledast_{s(1)} \in \Omega_{s(1)}, \circledast_{s(2)} \in \Omega_{s(2)}$ найдется такая операция $\circledast_{s(3)} \in \Omega_{s(3)}$, что тройка $\left\langle\circledast_{1}, \circledast_{2}, \circledast_{3}\right\rangle$ инцидентна.

Лемма 5.2. Пусть $\left\langle\Omega_{1}, \Omega_{2}, \Omega_{3}\right\rangle$ - I-система и множества $\Omega_{1}, \Omega_{2}, \Omega_{3}$ не nустьл. Тогда существует такая групповая операџия + на множестве $B$, что каждая операџиия из множества $\Omega_{1} \cup \Omega_{2} \cup \Omega_{3}$ изотопна операџии +.

Доказательство. Выберем операции $\circledast_{1} \in \Omega_{1}$, $\circledast_{2} \in \Omega_{2}$. По определению существует такая операция $\circledast_{3} \in \Omega_{3}$, что тройка $\left\langle\circledast_{1}, \circledast_{2}, \circledast_{3}\right\rangle$ инцидентна. В силу леммы 5.1 существует такая групповая операция + , что операции $\circledast_{1}, \circledast_{2}, \circledast_{3}$ ей изотопны. 
Выберем еще одну операцию $\odot_{1} \in \Omega_{1}$. Опять же по определению существует такая операция $\odot_{3} \in \Omega_{3}$, что тройка $\left\langle\odot_{1}, \circledast_{2}, \odot_{3}\right\rangle$ инцидентна, и в силу леммы 5.1 существует такая групповая операция $\oplus$, что операции $\odot_{1}, \circledast_{2}, \odot_{3}$ ей изотопны.

Таким образом, групповые операции,$+ \oplus$ изотопны операции $\circledast_{2}$. Так как отношение изотопии является отношением эквивалентности, то операции ,$+ \oplus$ изотопны. Следовательно, операции,$+ \odot_{1}$ изотопны операции $\oplus$, откуда следует, что операции,$+ \odot_{1}$ изотопны друг другу. Так как операция $\odot_{1} \in \Omega_{1}$ выбиралась произвольно, то все операции из множества $\Omega_{1}$ изотопны операции + .

Аналогично показываем, что все операции из множеств $\Omega_{2}$ и $\Omega_{3}$ изотопны операции + .

Лемма 5.3. Пусть $\left\langle\Omega_{1}, \Omega_{2}, \Omega_{3}\right\rangle$ - I-система, множества $\Omega_{1}, \Omega_{2}, \Omega_{3}$ не пусты и + - такая групповая операџия на множестве $B$, что каждая операция из множества $\Omega_{1} \cup \Omega_{2} \cup \Omega_{3}$ ей изотопна. Тогда существуют такие подстановки $\alpha_{1}, \alpha_{2}, \alpha_{3} \in \Sigma_{B}^{(0)}$, что

$$
\Omega_{1} \subseteq \mathcal{H}\left(+,-\alpha_{2}, \alpha_{3}\right), \quad \Omega_{2} \subseteq \mathcal{H}\left(+,-\alpha_{1}, \alpha_{3}\right), \quad \Omega_{3} \subseteq \mathcal{H}\left(+,-\alpha_{1}, \alpha_{2}\right) .
$$

Доказательство. Выберем произвольно операции $\circledast_{3} \in \Omega_{3}, \circledast_{2} \in \Omega_{2}$. В качестве $\circledast_{1}$ возьмем такую операцию $\circledast_{1} \in \Omega_{1}$, что тройка $\left\langle\circledast_{1}, \circledast_{2}, \circledast_{3}\right\rangle$ инцидентна. В условиях леммы

$$
x \circledast_{i} y=\kappa_{i}^{-1}\left(\lambda_{i}(x)+\mu_{i}(y)\right),
$$

где $\lambda_{i}, \mu_{i} \in \Sigma_{B}^{(0)}, \kappa_{i} \in \Sigma_{B}$ (см. лемму 3.1).

Применив к тройке $\left\langle\circledast_{1}, \circledast_{2}, \circledast_{3}\right\rangle$ теорему 5.1, получим, что существуют такие автоморфизмы $f, h$ группы $\langle B ;+\rangle$, для которых выполняются равенства

$$
\mu_{2}=h \mu_{1}, \quad \lambda_{1}=-f^{-1} \mu_{3}, \quad \lambda_{3}=f^{-1} h \lambda_{2} .
$$

Положим

$$
\alpha_{1}=-\lambda_{3}, \quad \alpha_{2}=\mu_{3}, \quad \alpha_{3}=\mu_{2} .
$$

В этих обозначениях имеем

$$
\mu_{1}=h^{-1} \alpha_{3}, \quad \lambda_{1}=-f^{-1} \alpha_{2}, \quad \lambda_{2}=-h^{-1} f \alpha_{1} .
$$

Выберем еще одну произвольную операцию $\odot_{3} \in \Omega_{3}$. Она также представима в виде изотопа

$$
x \odot_{3} y=\kappa^{-1}(\lambda(x)+\mu(y)) .
$$


Существуют такие операции $\odot_{1} \in \Omega_{1}, \odot_{2} \in \Omega_{2}$, что тройки $\left\langle\odot_{1}, \circledast_{2}, \odot_{3}\right\rangle$, $\left\langle\circledast_{1}, \odot_{2}, \odot_{3}\right\rangle$ инцидентны. Применив к ним теорему 5.1 , получим, что существуют автоморфизмы $g, q$ группы $\langle B ;+\rangle$, для которых выполняются равенства

$$
\mu=-g \lambda_{1}=g f^{-1} \alpha_{2}, \quad \lambda=q \lambda_{2}=-q h^{-1} f \alpha_{1},
$$

т. е. $\odot_{3} \subseteq \mathcal{H}\left(+,-\alpha_{1}, \alpha_{2}\right)$. Так как операция $\odot_{3}$ выбиралась произвольно, то $\Omega_{3} \subseteq \mathcal{H}\left(+,-\alpha_{1}, \alpha_{2}\right)$.

Выбрав произвольно операцию $\odot_{2} \in \Omega_{2}$ и рассмотрев инцидентные тройки $\left\langle\odot_{1}, \odot_{2}, \circledast_{3}\right\rangle,\left\langle\circledast_{1}, \odot_{2}, \odot_{3}\right\rangle$, получим, что $\Omega_{2} \subseteq \mathcal{H}\left(+,-\alpha_{1}, \alpha_{3}\right)$.

Третье включение доказывается аналогично.

3 а м е ч а н и е. При доказательстве леммы мы указали конструктивный способ построения подстановок $\alpha_{i}$ : выбрать произвольно операции $\circledast{ }_{3} \in \Omega_{3}$, $\circledast_{2} \in \Omega_{2}$, рассмотреть одно из их представлений (5.7) в виде изотопа группь и определить подстановки равенствами (5.8).

В ряде случаев удобно применять другие способы, аналогичные рассмотренному. Например, можно выбрать операции $\circledast_{1} \in \Omega_{3}, \circledast_{2} \in \Omega_{2}$, pacсмотреть представление (5.7) и положить

$$
\alpha_{1}=-\lambda_{2}, \quad \alpha_{2}=\lambda_{1}, \quad \alpha_{3}=\mu_{2}
$$

или

$$
\alpha_{1}=-\lambda_{2}, \quad \alpha_{2}=\lambda_{1}, \quad \alpha_{3}=\mu_{1} .
$$

Лемма 5.4. Пусть

$$
\Omega_{1}=\mathcal{H}\left(+,-\alpha_{2}, \alpha_{3}, S_{1}\right), \Omega_{2}=\mathcal{H}\left(+,-\alpha_{1}, \alpha_{3}, S_{2}\right), \Omega_{3}=\mathcal{H}\left(+,-\alpha_{1}, \alpha_{2}, S_{3}\right)
$$

- непустые множества.

Тогда тройка $\left\langle\Omega_{1}, \Omega_{2}, \Omega_{3}\right\rangle$ является I-системой в том и только в том случае, когда существуют такая подгруппа $T \subseteq$ Aut $\langle B ;+\rangle$ и такие элементы $e_{1}, e_{3} \in \operatorname{Aut}\langle B ;+\rangle$, чmo $S_{1}=T e_{1}, S_{3}=e_{3} T, S_{2}=e_{3} T e_{1}$.

Доказательство. Рассмотрим произвольные операции $\circledast_{1} \in \Omega_{1}, \circledast_{2} \in \Omega_{2}$, $\circledast_{3} \in \Omega_{3}$. Учитывая равенство (3.1), их можно представить в виде

$$
\begin{aligned}
& x \circledast_{1} y=\kappa_{1}^{-1}\left(-\alpha_{2}(x)+h_{1} \alpha_{3}(y)\right), \\
& x \circledast_{2} y=\kappa_{2}^{-1}\left(-\alpha_{1}(x)+h_{2} \alpha_{3}(y)\right), \\
& x \circledast_{3} y=\kappa_{3}^{-1}\left(-\alpha_{1}(x)+h_{3} \alpha_{2}(y)\right),
\end{aligned}
$$

где $\kappa_{1}, \kappa_{2}, \kappa_{3} \in \Sigma_{B}, h_{1} \in S_{1}, h_{2} \in S_{2}, h_{3} \in S_{3}$. 
Теорема 5.1 показывает, что тройка $\left\langle\circledast_{1}, \circledast_{2}, \circledast_{3}\right\rangle$ инцидентна тогда и только тогда, когда найдутся такие автоморфизмы $f, q \in \operatorname{Aut}\langle B ;+\rangle$, что выполняются равенства

$$
\begin{aligned}
h_{2} \alpha_{3} & =q h_{1} \alpha_{3}, \\
h_{3} \alpha_{2} & =f \alpha_{2}, \\
-\alpha_{1} & =-f q^{-1} \alpha_{1} .
\end{aligned}
$$

Легко видеть, что если равенство

$$
h_{2}=h_{3} h_{1}
$$

не выполняется, то система равенств (5.9) не выполняется ни для каких автоморфизмов $f, q$. Если равенство (5.10) выполняется, то система равенств (5.9) выполняется при $q=f=h_{3}$.

Таким образом, рассматриваемая тройка $\left\langle\circledast_{1}, \circledast_{2}, \circledast_{3}\right\rangle$ инцидентна тогда и только тогда, когда выполняется равенство (5.10). Следовательно, тройка $\left\langle\Omega_{1}, \Omega_{2}, \Omega_{3}\right\rangle$ является $I$-системой тогда и только тогда, когда тройка $\left\langle S_{1}, S_{2}, S_{3}\right\rangle$ удовлетворяет следующему условию: для любой подстановки $s$ множества $\{1,2,3\}$ и любых элементов $h_{s(1)} \in S_{s(1)}, h_{s(2)} \in S_{s(2)}$ найдется такой элемент $h_{s(3)} \in S_{s(3)}$, что выполняется равенство (5.10). Это эквивалентно тому, что выполняются включения

$$
S_{1} \supseteq S_{3}^{-1} S_{2}, \quad S_{2} \supseteq S_{3} S_{1}, \quad S_{3} \supseteq S_{2} S_{1}^{-1}
$$

Легко видеть, что если $S_{1}=T e_{1}, S_{3}=e_{3} T, S_{2}=e_{3} T e_{1}$ для некоторой группы $T$, то включения (5.11) выполняются и тройка $\left\langle\Omega_{1}, \Omega_{2}, \Omega_{3}\right\rangle$ является $I$-системой.

Допустим, что включения (5.11) выполняются. Выберем произвольно элементы $e_{1} \in S_{1}, e_{3} \in S_{3}$. Положим $T=e_{3}^{-1} S_{2} e_{1}^{-1}$.

Пусть $g \in T$. Тогда $e_{3} g e_{1} \in S_{2}$ и $e_{3} g=\left(e_{3} g e_{1}\right) e_{1}^{-1} \in S_{2} S_{1}^{-1} \subseteq S_{3}$, т. е. $e_{3} T \subseteq S_{3}$. Обратно пусть $h \in S_{3}$. Тогда $h e_{1} \in S_{3} S_{1} \subseteq S_{2}, e_{3}^{-1} h=$ $e_{3}^{-1}\left(h e_{1}\right) e_{1}^{-1} \in T$ и $h=e_{3}\left(e_{3}^{-1} h\right) \in e_{3} T$. Таким образом, $e_{3} T=S_{3}$.

Аналогично показываем, что $S_{1}=T e_{1}$.

Пусть $g_{1}, g_{3} \in T$. Тогда произведение $\left(e_{3} g_{3}\right)\left(g_{1} e_{1}\right)$ принадлежит множеству $S_{2}$ и, следовательно, произведение $g_{3} g_{1}$ принадлежит множеству $T$. Таким образом, множество $T$ является группой. 


\section{GA-решетки длины 3 и более}

Исследуем неразложимые $G A$-решетки длины 3 и более. Следствие 4.2 и лемма 1.5 показывают, что каждая такая решетка изоморфна $G A$-решетке отношений эквивалентности на множестве $B^{n}$ для некоторого конечного множества $B$.

Рассмотрим множество $B^{n}$, где $n \geqslant 3$, и неразложимую $G A$-решетку $\mathbf{L}_{B^{n}}$ отношений эквивалентности на множестве $B^{n}$. Будем использовать обозначения, введенные в разделе 4 . В частности, там определены $G A$-решетки $\mathbf{L}_{\{i, j\}}$ отношений эквивалентности на множестве $B^{2}$. Из теоремы 2.3 следует, что

$$
\mathbf{L}_{\{i, j\}}=\mathbf{L}_{B^{2}}\left(\Omega_{\{i, j\}} \cup\left\{\diamond_{r}, \diamond_{l}\right\}\right)
$$

для слабо ортогонального $\Sigma_{B}$-подмножества $\Omega_{\{i, j\}} \subseteq \mathcal{Q}(B)$. Из определений следует равенство

$$
\mathbf{L}_{\{i, j\}}^{*}=\left\langle\operatorname{ker} \circledast \mid \circledast \in \Omega_{\{i, j\}}\right\rangle .
$$

Согласно теореме 4.4 все множества $\Omega_{\{i, j\}}$ не пусты.

Следствие 6.1. Пусть $i_{1}<i_{2}<i_{3}$. Тогда тройка

$$
\left\langle\Omega_{\left\{i_{2}, i_{3}\right\}}, \Omega_{\left\{i_{1}, i_{3}\right\}}, \Omega_{\left\{i_{1}, i_{2}\right\}}\right\rangle
$$

является I-системой.

Доказательство. Для простоты обозначений будем считать, что $i_{1}=1, i_{2}=$ $2, i_{3}=3$.

Выберем произвольные операции $\circledast_{1} \in \Omega_{\{2,3\}}, \circledast_{2} \in \Omega_{\{1,3\}}$. В силу теоремы 4.1 элемент

$$
\tau=\pi_{Z_{n} \backslash\{1,2,3\}}^{(n)} \wedge\left(\zeta_{\{2,3\}}\left(\operatorname{ker} \circledast_{1}\right) \wedge \zeta_{\{1,3\}}\left(\operatorname{ker} \circledast_{2}\right)\right)
$$

является атомом решетки $\mathbf{L}_{B^{n}}$. В силу теоремы 4.2 существует такая операция $\circledast_{3} \in \Omega_{\{1,2\}}$, что

$$
\tau=\pi_{Z_{n} \backslash\{1,2,3\}}^{(n)} \wedge\left(\zeta_{\{2,3\}}\left(\operatorname{ker} \circledast_{1}\right) \wedge \zeta_{\{1,3\}}\left(\operatorname{ker} \circledast_{2}\right) \wedge \zeta_{\{1,2\}}\left(\operatorname{ker} \circledast_{3}\right)\right) .
$$

Из последнего равенства следует соотношение

$$
\tau \subseteq \pi_{Z_{n} \backslash\{1,2,3\}}^{(n)} \wedge\left(\zeta_{\{1,3\}}\left(\text { ker } \circledast_{2}\right) \wedge \zeta_{\{1,2\}}\left(\text { ker } \circledast_{3}\right)\right) .
$$


Так как обе части неравенства являются атомами, то они равны, значит,

$$
\begin{gathered}
\pi_{Z_{n} \backslash\{1,2,3\}}^{(n)} \wedge\left(\zeta_{\{2,3\}}\left(\operatorname{ker} \circledast_{1}\right) \wedge \zeta_{\{1,3\}}\left(\text { ker } \circledast_{2}\right) \wedge \zeta_{\{1,2\}}\left(\text { ker } \circledast_{3}\right)\right)= \\
=\pi_{Z_{n} \backslash\{1,2,3\}}^{(n)} \wedge\left(\zeta_{\{1,3\}}\left(\text { ker } \circledast_{2}\right) \wedge \zeta_{\{1,2\}}\left(\text { ker } \circledast_{3}\right)\right) .
\end{gathered}
$$

Применив лемму 1.4 , получим

$\zeta_{\{2,3\}}\left(\operatorname{ker} \circledast_{1}\right) \wedge \zeta_{\{1,3\}}\left(\operatorname{ker} \circledast_{2}\right) \wedge \zeta_{\{1,2\}}\left(\operatorname{ker} \circledast_{3}\right)=\zeta_{\{1,3\}}\left(\operatorname{ker} \circledast_{2}\right) \wedge \zeta_{\{1,2\}}\left(\operatorname{ker} \circledast_{3}\right)$, откуда следует, что

$$
\zeta_{\{1,3\}}\left(\operatorname{ker} \circledast_{2}\right) \wedge \zeta_{\{1,2\}}\left(\operatorname{ker} \circledast_{3}\right) \subseteq \zeta_{\{2,3\}}\left(\operatorname{ker} \circledast_{1}\right) .
$$

Сравнив это неравенство с формулами (5.1) и (5.2), получаем, что тройка $\left\langle\circledast_{1}, \circledast_{2}, \circledast_{3}\right\rangle$ инцидентна.

Аналогично рассматриваются случаи, когда выбраны две другие операции из тройки $\left\langle\circledast_{1}, \circledast_{2}, \circledast_{3}\right\rangle$.

Следствие 6.2. Все операции из множества $\bigcup_{1 \leqslant i<j \leqslant n} \Omega_{\{i, j\}}$ изотопны некоторой групповой операции.

Доказательство. Применив следствие 6.1 и лемму 5.2 к тройке $\{1,2,3\}$, получаем, что все операции из множества $\Omega_{\{1,2\}}$ изотопны некоторой групповой операции + . Из тех же утверждений, примененных к тройке $\{1,2, m\}(m \geqslant 3)$, получаем, что все операции из множества $\Omega_{\{1, m\}}$ изотопны всем операциям из множества $\Omega_{\{1,2\}}$. Так как отношение изотопии является отношением эквивалентности, то все операции из множества $\Omega_{\{1, m\}}$ изотопны той же операции + .

Пусть $1<i<j$. Тогда те же утверждения показывают, что все операции из множества $\Omega_{\{i, j\}}$ изотопны всем операциям из множества $\Omega_{\{1, i\}}$ и, следовательно, изотопны операции + .

Допустим, что рассматриваемое множество $B$ является носителем векторного пространства $\mathbf{B}=\langle B ;+, \mathbb{F}\rangle, \vec{\alpha}=\left(\alpha_{1}, \ldots, \alpha_{n}\right)$ - семейство подстановок, принадлежащих множеству $\Sigma_{B}^{(0)}$. Через $\mathcal{F}_{\vec{\alpha}}\langle B ;+, \mathbb{F}\rangle$ обозначим множество всех форм $\sum_{i=1}^{n} c_{i} \alpha_{i}\left(x_{i}\right), c_{i} \in \mathbb{F}$, определенных на множестве $B^{n}$. Множество всех отношений, являющихся пересечением какого-либо множества ядер таких форм, обозначим $\mathcal{L}_{\vec{\alpha}}\langle B ;+, \mathbb{F}\rangle$.

Теорема 6.1. Пусть $\mathbf{L}_{B^{n}}$ - неразложимая GA-решетка отношений эквивалентности на множестве $B^{n}$ u $n \geqslant 3$. Тогда на множестве $B$ можно так определить структуру векторного пространства над полем $\mathbb{F}$, что $\mathbf{L}_{B^{n}}=\mathcal{L}_{\vec{\alpha}}\langle B ;+, \mathbb{F}\rangle$ для подходящих подстановок $\alpha_{1}, \ldots, \alpha_{n} \in \Sigma_{B}^{(0)}$. 
Доказательство. В силу следствия 6.2 существует такая групповая операция + на множестве $B$, что все операции из каждого множества $\Omega_{\{i, j\}}$ ей изотопны.

Произвольно выберем операции $\circledast_{m} \in \Omega_{\{1, m\}}$. В силу выбора операции + и леммы 3.1 для подходящих подстановок $\lambda_{m}, \mu_{m} \in \Sigma_{B}^{(0)}, \kappa_{m} \in \Sigma_{B}$ выполняются равенства ${ }_{\lambda_{m}}^{\kappa_{m}}+\mu_{m} \in \Omega_{\{1, m\}}$.

Применив следствие 6.1, леммы 5.3, 3.3 и 5.4 к тройке $\{1,2, m\}$, получим, что

$$
\begin{aligned}
\Omega_{\{2, m\}} & =\mathcal{H}\left(+,-\alpha_{2}, \alpha_{m}, T^{(m)} e_{1}^{(m)}\right), \\
\Omega_{\{1, m\}} & =\mathcal{H}\left(+,-\alpha_{1}, \alpha_{m}, e_{3}^{(m)} T^{(m)} e_{1}^{(m)}\right), \\
\Omega_{\{1,2\}} & =\mathcal{H}\left(+,-\alpha_{1}, \alpha_{2}, e_{3}^{(m)} T^{(m)}\right) .
\end{aligned}
$$

Здесь $T^{(m)}-$ подгруппа группы $\operatorname{Aut}\langle B ;+\rangle, e_{j}^{(m)} \in \operatorname{Aut}\langle B ;+\rangle, \alpha_{1}=-\lambda_{2}$, $\alpha_{k}=\mu_{k}(k \geqslant 2)$.

Из определений следует, что ${ }_{-\alpha_{1}}^{\kappa_{2}}+\alpha_{2} \in \mathcal{H}\left(+,-\alpha_{1}, \alpha_{2}, e_{3}^{(m)} T^{(m)}\right)$ тогда и только тогда, когда $1_{B} \in e_{3}^{(m)} T^{(m)}$, что выполняется тогда и только тогда, когда $e_{3}^{(m)} \in T^{(m)}$. В этом случае $e_{3}^{(m)} T^{(m)}=T^{(m)}$ и элемент $e_{3}^{(m)}$ можно удалить из формул (6.1).

Теперь

$$
{ }_{-\alpha_{1}}^{\kappa_{m}}+{ }_{\alpha_{m}} \in \mathcal{H}\left(+,-\alpha_{1}, \alpha_{2}, T^{(m)} e_{1}^{(m)}\right),
$$

отсюда так же, как выше, получаем равенство $T^{(m)} e_{1}^{(m)}=T^{(m)}$, поэтому элемент $e_{1}^{(m)}$ также можно удалить.

Далее,

$$
\mathcal{H}\left(+,-\alpha_{1}, \alpha_{2}, T^{(m)}\right)=\mathcal{H}\left(+,-\alpha_{1}, \alpha_{2}, T^{(3)}\right) .
$$

Из определений следует, что последнее равенство выполняется тогда и только тогда, когда $T^{(m)}=T^{(3)}$ для всех $m$. В дальнейшем группу $T^{(3)}$ будем обозначать через $T$, что дает выражение

$$
\Omega_{\{1, m\}}=\mathcal{H}\left(+,-\alpha_{1}, \alpha_{m}, T\right) .
$$

Применив те же результаты к тройке $\{1, i, j\}$, где $1<i<j$, получим с учетом замечания, приведенного после доказательства леммы 5.3, что

$$
\Omega_{\{i, j\}}=\mathcal{H}\left(+,-\alpha_{i}, \alpha_{j}, T\right) .
$$


Пусть $h_{2}, h_{3} \in T$. Тогда операция $\circledast 1 j={ }_{-\alpha_{1}}+h_{j} \alpha_{j}$ принадлежит множеству $\Omega_{\{1, j\}}$. Следовательно, каждое отношение $\mathrm{ker} \circledast{ }_{1 j}$ принадлежит решетке $\mathbf{L}_{\{1, j\}}$, а отношения

$$
\theta_{2}=\zeta_{\{1,2\}}\left(\operatorname{ker} \circledast_{12}\right) \wedge \pi_{3}^{(n)}, \quad \theta_{3}=\zeta_{\{1,3\}}\left(\operatorname{ker} \circledast_{13}\right) \wedge \pi_{2}^{(n)}
$$

принадлежат решетке $\mathbf{L}_{B^{n}}$. Вычислим их объединение $\rho_{h_{2}, h_{3}}$. Так как отношения $\theta_{2}, \theta_{3}$ перестановочны, то $\rho_{h_{2}, h_{3}}=\theta_{2} \circ \theta_{3}$, и нам достаточно вычислить произведение рассматриваемых отношений.

Рассмотрим произвольные векторы $\mathbf{a}=\left(a_{1}, \ldots, a_{n}\right), \mathbf{b}=\left(b_{1}, \ldots, b_{n}\right) \in$ $B^{n}$. В соответствии с определением $(\mathbf{a}, \mathbf{b}) \in \theta_{2} \circ \theta_{3}$ тогда и только тогда, когда существует такой вектор $\mathbf{x}=\left(x_{1}, \ldots, x_{n}\right)$, что

$$
\left\{\begin{aligned}
-\alpha_{1}\left(x_{1}\right)+h_{2} \alpha_{2}\left(x_{2}\right) & =-\alpha_{1}\left(a_{1}\right)+h_{2} \alpha_{2}\left(a_{2}\right), \\
x_{3} & =a_{3}, \\
-\alpha_{1}\left(x_{1}\right)+h_{3} \alpha_{3}\left(x_{3}\right) & =-\alpha_{1}\left(b_{1}\right)+h_{3} \alpha_{3}\left(b_{3}\right), \\
x_{2} & =b_{2} .
\end{aligned}\right.
$$

Другими словами, $(\mathbf{a}, \mathbf{b}) \in \theta_{2} \circ \theta_{3}$ тогда и только тогда, когда система уравнений (6.2) относительно неизвестных $x_{1}, x_{2}, x_{3}$ совместна. Нетрудно видеть, что неизвестные $x_{2}, x_{3}$ однозначно определяются, а первое и третье уравнения дают два выражения для неизвестного $x_{1}$. Ясно, что система совместна тогда и только тогда, когда значения этих выражений совпадают, т. е. выполняется равенство

$$
-\alpha_{1}\left(a_{1}\right)+h_{2} \alpha_{2}\left(a_{2}\right)-h_{2} \alpha_{2}\left(b_{2}\right)=-\alpha_{1}\left(b_{1}\right)+h_{3} \alpha_{3}\left(b_{3}\right)-h_{3} \alpha_{3}\left(a_{3}\right),
$$

которое можно записать в эквивалентном виде:

$$
h_{3} \alpha_{3}\left(a_{3}\right)-h_{3} \alpha_{3}\left(b_{3}\right)+\alpha_{1}\left(b_{1}\right)-\alpha_{1}\left(a_{1}\right)+h_{2} \alpha_{2}\left(a_{2}\right)-h_{2} \alpha_{2}\left(b_{2}\right)=0 .
$$

Получили явное описание отношения $\rho_{h_{2}, h_{3}}=\theta_{2} \vee \theta_{3}$ : векторы $\left(a_{1}, \ldots, a_{n}\right)$, $\left(b_{1}, \ldots, b_{n}\right)$ находятся в отношении $\rho_{h_{2}, h_{3}}$ тогда и только тогда, когда выполняется равенство (6.3).

Отношение $\theta_{2} \vee \theta_{3}$ является отношением эквивалентности. В частности, это означает, что равенство (6.3) выполняется тогда и только тогда, когда выполняется «симметричное» равенство

$$
h_{3} \alpha_{3}\left(b_{3}\right)-h_{3} \alpha_{3}\left(a_{3}\right)+\alpha_{1}\left(a_{1}\right)-\alpha_{1}\left(b_{1}\right)+h_{2} \alpha_{2}\left(b_{2}\right)-h_{2} \alpha_{2}\left(a_{2}\right)=0 .
$$

Произвольно выберем элементы $d_{1}, d_{2} \in B$. Положим

$$
\begin{gathered}
a_{1}=\alpha_{1}^{-1}\left(d_{1}\right), \quad a_{2}=\left(h_{2} \alpha_{2}\right)^{-1}\left(-d_{2}\right), \quad a_{3}=\left(h_{3} \alpha_{3}\right)^{-1}\left(d_{2}+d_{1}\right), \\
b_{1}=b_{2}=b_{3}=0 .
\end{gathered}
$$


Подставив эти значения в формулу (6.3), получим

$$
\begin{gathered}
h_{3} \alpha_{3}\left(\left(h_{3} \alpha_{3}\right)^{-1}\left(d_{2}+d_{1}\right)\right)-h_{3} \alpha_{3}(0)+\alpha_{1}(0)-\alpha_{1}\left(\alpha_{1}^{-1}\left(d_{1}\right)\right)+ \\
+h_{2} \alpha_{2}\left(\left(h_{2} \alpha_{2}\right)^{-1}\left(-d_{2}\right)\right)-h_{2} \alpha_{2}(0)=d_{2}+d_{1}-d_{1}-d_{2}=0,
\end{gathered}
$$

т. е. равенство (6.3) выполняется. Следовательно, для этих значений должно выполняться и равенство (6.4). Таким образом,

$$
\begin{aligned}
0= & h_{3} \alpha_{3}(0)-h_{3} \alpha_{3}\left(\left(h_{3} \alpha_{3}\right)^{-1}\left(d_{2}+d_{1}\right)\right)+\alpha_{1}\left(\alpha_{1}^{-1}\left(d_{1}\right)\right)-\alpha_{1}(0)+ \\
& +h_{2} \alpha_{2}(0)-h_{2} \alpha_{2}\left(\left(h_{2} \alpha_{2}\right)^{-1}\left(-d_{2}\right)\right)=-\left(d_{2}+d_{1}\right)+d_{1}+d_{2},
\end{aligned}
$$

и $d_{2}+d_{1}=d_{1}+d_{2}$. Так как элементы $d_{1}, d_{2}$ выбирались произвольно, то группа $\langle B ;+\rangle$ абелева.

Для абелевой группы $\langle B ;+\rangle$ условие (6.3) эквивалентно условию

$$
-\alpha_{1}\left(a_{1}\right)+h_{2} \alpha_{2}\left(a_{2}\right)+h_{3} \alpha_{3}\left(a_{3}\right)=-\alpha_{1}\left(b_{1}\right)+h_{2} \alpha_{2}\left(b_{2}\right)+h_{3} \alpha_{3}\left(b_{3}\right),
$$

которое и определяет отношение $\rho_{h_{2}, h_{3}}$.

Пусть $g \in T$. Рассмотрим отношение $\sigma_{g}=\zeta_{23}\left(\operatorname{ker}\left(-\alpha_{2}+g \alpha_{3}\right)\right)$ и отношение $\eta=\left(\rho_{h_{2}, h_{3}} \wedge \sigma_{g}\right) \vee \pi_{\{1,2\}}^{(n)}$. Опять воспользовавшись тем, что решетка $\mathbf{L}_{B^{n}}$ перестановочна, получим $\eta=\left(\rho_{h_{2}, h_{3}} \wedge \sigma_{g}\right) \circ \pi_{\{1,2\}}^{(n)}$. Поэтому векторы $\mathbf{a}, \mathbf{b}$ находятся в отношении $\eta$ тогда и только тогда, когда совместна система уравнений

$$
\left\{\begin{aligned}
-\alpha_{1}\left(a_{1}\right)+h_{2} \alpha_{2}\left(a_{2}\right)+h_{3} \alpha_{3}\left(a_{3}\right) & =-\alpha_{1}\left(x_{1}\right)+h_{2} \alpha_{2}\left(x_{2}\right)+h_{3} \alpha_{3}\left(x_{3}\right), \\
-\alpha_{2}\left(a_{2}\right)+g \alpha_{3}\left(a_{3}\right) & =-\alpha_{2}\left(x_{2}\right)+g \alpha_{3}\left(x_{3}\right), \\
x_{1} & =b_{1}, \\
x_{2} & =b_{2} .
\end{aligned}\right.
$$

Последние три уравнения однозначно определяют неизвестные значения:

$$
\begin{gathered}
x_{1}=b_{1}, \quad x_{2}=b_{2}, \\
x_{3}=\alpha_{3}^{-1} g^{-1}\left(\alpha_{2}(b)-\alpha_{2}\left(a_{2}\right)+g \alpha_{3}\left(a_{3}\right)\right) .
\end{gathered}
$$

Подставив эти значения в первое уравнение, получим цепочку равенств

$$
\begin{aligned}
& -\alpha_{1}\left(a_{1}\right)+h_{2} \alpha_{2}\left(a_{2}\right)+h_{3} \alpha_{3}\left(a_{3}\right)= \\
& \quad=-\alpha_{1}\left(b_{1}\right)+h_{2} \alpha_{2}\left(b_{2}\right)+h_{3} \alpha_{3}\left(\alpha_{3}^{-1} g^{-1}\left(\alpha_{2}\left(b_{2}\right)-\alpha_{2}\left(a_{2}\right)+g \alpha_{3}\left(a_{3}\right)\right)\right)= \\
& \quad=-\alpha_{1}\left(b_{1}\right)+h_{2} \alpha_{2}\left(b_{2}\right)+h_{3} g^{-1} \alpha_{2}\left(b_{2}\right)-h_{3} g^{-1} \alpha_{2}\left(a_{2}\right)+h_{3} \alpha_{3}\left(a_{3}\right),
\end{aligned}
$$

дающую равенство

$$
-\alpha_{1}\left(a_{1}\right)+\left(h_{2}+h_{3} g^{-1}\right) \alpha_{2}\left(a_{2}\right)=-\alpha_{1}\left(b_{1}\right)+\left(h_{2}+h_{3} g^{-1}\right) \alpha_{2}\left(b_{2}\right) .
$$


Отношение $\zeta_{\{1,2\}}^{-1}(\eta)$ принадлежит решетке $\mathbf{L}_{\{1,2\}}$. Поэтому либо $\zeta_{\{1,2\}}^{-1}(\eta) \in \mathbf{D}^{(2)}\left(B^{2}\right)$, либо $\zeta_{\{1,2\}}^{-1}(\eta) \in \mathbf{L}_{\{1,2\}}^{*}$. В первом случае отображение $h_{2}+h_{3} g^{-1}$ является нулевым эндоморфизмом $O_{B}$. Во втором отображение $h_{2}+h_{3} g^{-1}$ равно некоторому автоморфизму $f \in T$.

Так как автоморфизмы $h_{2}+h_{3} g^{-1}$ выбирались произвольно, то множество $\mathbb{F}=T \cup\left\{O_{B}\right\}$ замкнуто относительно операции + и, следовательно, является полем, а множество $B$ - векторным пространством над этим полем.

Bсе отношения, входящие в решетку $\mathcal{L}_{\vec{\alpha}}\langle B ;+, \mathbb{F}\rangle$, перестановочны (см. [8]). Легко проверяется, что и другие свойства, входящие в определение $G A$-решеток, также выполняются и $\mathcal{L}_{\vec{\alpha}}\langle B ;+, \mathbb{F}\rangle$ является $G A$-решеткой.

Рассмотрим отношение

$$
\theta \in\left[\pi_{\{i, j\}}^{(2)}, \nabla_{B^{n}}\right]_{\mathcal{E}\left(B^{n}\right)} .
$$

Допустим, что $\theta \in \mathbf{L}_{B^{n}}$. Тогда либо $\theta \in \mathbf{D}^{(n)}\left(B^{n}\right)$, либо $\theta \in \Omega_{\{i, j\}}$. В первом случае $\theta \in \mathcal{L}_{\vec{\alpha}}\langle B ;+, \mathbb{F}\rangle$ в силу леммы 1.2. Во втором отношение $\theta$ имеет вид $\zeta_{\{i, j\}}\left(\operatorname{ker}\left({ }_{-g \alpha_{i}}^{\kappa}+g c \alpha_{j}\right)\right)$. Из равенств (3.1) и (1.5) следует, что отношение $\theta$ является ядром формы $\left(-1_{B}\right)\left(-\alpha_{i}\left(x_{i}\right)\right)+c \alpha_{j}\left(x_{j}\right)$, т. е. оно принадлежит решетке $\mathcal{L}_{\vec{\alpha}}\langle B ;+, \mathbb{F}\rangle$.

Допустим, что, напротив, $\theta \in \mathcal{L}_{\vec{\alpha}}\langle B ;+, \mathbb{F}\rangle$. Тогда $\theta=\operatorname{ker}\left(\sum_{i=1}^{n} c_{i} \alpha_{i}\left(x_{i}\right)\right)$ для подходящих элементов $c_{1}, \ldots, c_{n} \in \mathbb{F}$. Если хотя бы один из элементов $c_{k}(k \notin\{i, j\})$ отличен от $O_{B}$, то неравенство $\theta \supseteq \pi_{\{i, j\}}^{(n)}$ не выполняется, что противоречит условию. Поэтому все эти элементы равны 0 .

Если $c_{i}=0$ или $c_{j}=0$, то $\theta \in \mathbf{D}^{(n)}\left(B^{n}\right)$ и $\theta \in \mathbf{L}_{B^{n}}$.

Осталось рассмотреть случай, когда $c_{i}, c_{j} \neq 0$. Имеем

$$
c_{i} \alpha_{i}\left(x_{i}\right)+c_{j} \alpha_{j}\left(x_{j}\right)=x_{i}\left(\begin{array}{l}
-c_{i} \\
-\alpha_{i}
\end{array}+\left(-c_{i}^{-1} c_{j}\right) \alpha_{j}\right) x_{j},
$$

откуда следует, что

$$
\theta=\zeta_{\{i, j\}}\left(\operatorname{ker}\left(\begin{array}{l}
-c_{i} \\
-\alpha_{i}
\end{array}+\left(-c_{i}^{-1} c_{j}\right) \alpha_{j}\right)\right),
$$

и опять же $\theta \in \mathbf{L}_{B^{n}}$.

Таким образом, следы решеток $\mathbf{L}_{B^{n}}$ и $\mathcal{L}_{\vec{\alpha}}\langle B ;+, \mathbb{F}\rangle$ в каждом из множеств $B_{i} \times B_{j}$, где $B_{i}, B_{j}-$ копии множества $B$, одинаковы. Результаты раздела 4 показывают, что множество таких следов однозначно определяет $G A$-решетку. Поэтому $\mathbf{L}_{B^{n}}=\mathcal{L}_{\vec{\alpha}}\langle B ;+, \mathbb{F}\rangle$. 


\section{Список литературы}

[1] Белоусов В. Д., Основы теории квазигрупn и луn, М.: Наука, 1967, 224 с.

[2] Белоусов В. Д., “Системы квазигрупп с обобщенными тождествами”, Успехи матем. наук, $\mathbf{X X}: 1(121), 75-146$.

[3] Глухов М. М., “О методах построения систем ортогональных квазигрупп с использованием групп”, Математические вопросы криптографии, $2: 4$ (2011), 5-24.

[4] Горчинский Ю.Н., “О гомоморфизмах многоосновных универсальных алгебр в связи с криптографическими применениями”, Труды по дискретной математике, 1, М.: Научное изд-во ТВП, 1997, 67-84.

[5] Гретцер Г., Общая теория решеток, М. : Мир, 1982, 456 с.

[6] Кон П., Универсальная алгебра, М.: Мир, 1968, 359 с.

[7] Курош А. Г., Лекичии по общей алгебре, М.: Наука, 1973, 40 с.

[8] Мальцев А.И., “К общей теории алгебраических систем”, Матем. сб. (новая серия), 35(77): 1 (1954), 3-20.

[9] Полин С. В., "Решение уравнений методом последовательного группирования и его оптимизация", Математические вопросы криптографии, 3:1 (2012), 97-123.

[10] Полин С. В., “Системы уравнений и решетки конгруэнций универсальных алгебр”, Математические вопросы криптографии, 4:4 (2013), 109-144.

[11] Полин С. В., “Перестановочные решетки отношений эквивалентности декартовых произведений”, Математические вопросы криптографии, 5:3 (2014), 81-116.

[12] Полин С. В., "Перестановочные решетки отношений эквивалентности на декартовых произведениях и согласованные с ними системы уравнений. I”, Математические вопросы криптографии, 6:1 (2015), 135-158.

[13] Харари Ф., Теория графов, М.: Мир, 1973, 300 с. 